\title{
Intersection Safety using Lidar and Stereo Vision sensors
}

\author{
Olivier Aycard*, Qadeer Baig*, Siviu Bota ${ }^{\ddagger}$, Fawzi Nashashibi ${ }^{\dagger}$, Sergiu Nedevschi $^{\ddagger}$, \\ Cosmin Pantilie ${ }^{\ddagger}$, Michel Parent ${ }^{\dagger}$, Paulo Resende ${ }^{\dagger}$, Trung-Dung $\mathrm{Vu}^{*}$ \\ *University of Grenoble1 - FRANCE \\ Email: FirstName.LastName@imag.fr \\ ${ }^{\dagger}$ INRIA Rocquencourt - FRANCE \\ Email: FirstName.LastName@inria.fr \\ $\ddagger$ Technical University of Cluj-Napoca - ROMANIA \\ Email: FirstName.LastName@cs.utcluj.ro
}

\begin{abstract}
In this paper, we describe our approach for intersection safety developed in the scope of the European project INTERSAFE-2. A complete solution for the safety problem including the tasks of perception and risk assessment using on-board lidar and stereo-vision sensors will be presented and interesting results are shown.
\end{abstract}

\section{INTRODUCTION}

About $30 \%$ to $60 \%$ (depending on the country) of all injury accidents and about $16 \%$ to $36 \%$ of the fatalities are intersection related. In addition, accident scenarios at intersections are amongst the most complex (different type of road users, various orientations and speeds).

The INTERSAFE- 2 project ${ }^{1}$ aims to develop and demonstrate a Cooperative Intersection Safety System (CISS) that is able to significantly reduce injury and fatal accidents at intersections. Vehicles equipped with communication means and onboard sensor systems cooperate with the road side infrastructure in order to achieve a comprehensive system that contributes to the EU-25 and zero accident vision as well as to a significant improvement of efficiency in traffic flow and thus reduce fuel consumption in urban areas. By networking state-of-the-art technologies for sensors, infrastructure systems, communications, digital map contents and new accurate positioning techniques, INTERSAFE-2 aims to bring Intersection Safety Systems much closer to market introduction. This paper aims to detail the technical solution developed on the Volkswagen demonstrator of the project. This solution takes as inputs raw data from a lidar and a stereo-vision system and delivers as an output a level of risk between the host vehicle and other entities present at the intersection. This paper is a joint paper between: INRIA Rocquencourt (France), Technical University of Cluj (Romania) and University of Grenoble1 (France).

The rest of the paper is organized as follows. In the next section, we present the demonstrator used for this work and sensors installed on it. We summarize the software architecture in section III. In sections IV \& V we present the sensor processing of lidar and stereo-vision. In section VI and VII, we detail our work on fusion and tracking. The Risk

${ }^{1}$ http://www.intersafe-2.eu

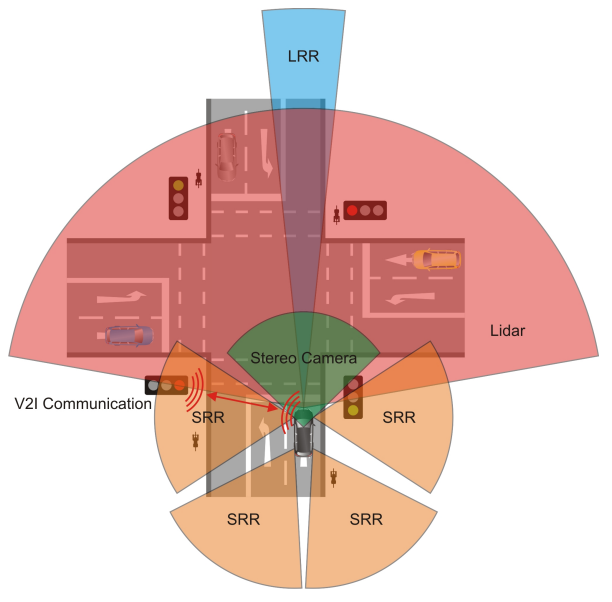

Fig. 1. Sensors installed on the demonstrator vehicle

Assessment module is described in section VIII. Experimental results are reported in section IX. We conclude this work in section $\mathrm{X}$.

\section{EXPERIMENTAL SETUP}

The demonstrator vehicle used to get datasets for this work has multiple sensors installed on it. It has a long range laser scanner with a field of view of $160^{\circ}$ and a maximum range of $150 \mathrm{~m}$. Other sensors installed on this demonstrator include a stereo vision camera, four short range radars (SRR) one at each corner of the vehicle and a long range radar (LRR) in front of the vehicle (Figure 1). Our work presented in this paper is only concerned with the processing and fusion of lidar and stereo vision data.

\section{SOFTWARE ARCHITECTURE}

Figure 2 illustrates the software architecture of the system. This architecture is composed of 5 modules:

1) The lidar data processing module which takes as input the raw data provided by the laser scanner and delivers (i) an estimation of the position of the host vehicle in the intersection and an estimation of its speed and (ii) a list of detected objects with their respective states (static or dynamic). An object is defined by the front line segment of the object (the visible part) and the 


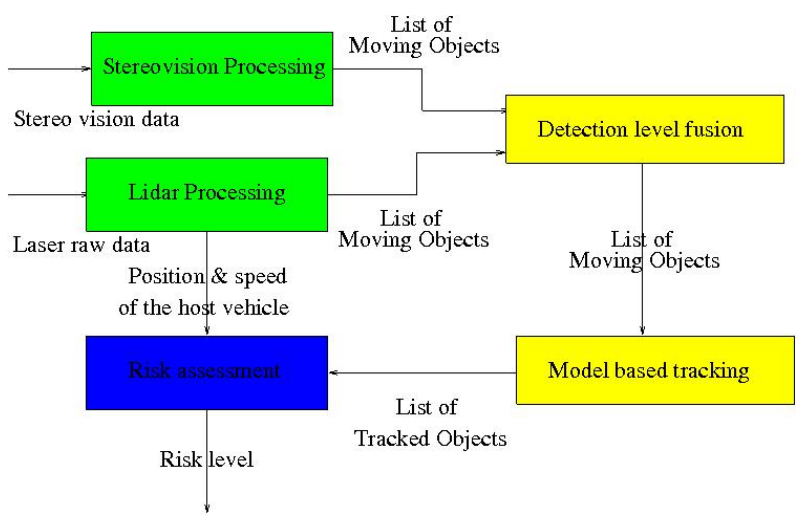

Fig. 2. Software architecture of the system

middle point of this segment. This module has been developed by University of Grenoble 1;

2) The stereo-vision data processing module which takes as input the raw data provided by the two camera and delivers as output a list of detected objects with their class (pedestrian, car or pole). An object is defined similarly to objects detected by the lidar in order to ease the fusion process. This module has been developed by Technical University of Cluj;

3) The fusion module takes as input the list of the detected objects provided by both kind of sensors and delivers a fused list of detected objects. For each object we have the front line segment of the object, the middle point of this segment, the class of the object and the number of sensors that have detected the object. This module has been developed by University of Grenoble 1 .

4) The tracking module takes as input the fused list of laser and stereo-vision objects and delivers a list of tracked objects. This module has been developed by University of Grenoble 1;

5) The risk assessment module which takes as inputs (i) the position and speed of the host vehicle and (ii) the list of tracked objects and delivers an estimation of the collision risk between the host vehicle and objects present in the environment. This module has been developed by INRIA Rocquencourt.

Each module will be described in more detail in the following.

\section{Lidar PRocessing}

We summarize our lidar data processing that we have used for moving objects detection with laser data (more detail can be found in [2]. This process consists of following steps: first we construct a local grid map and localize the vehicle in this map, then using this map we classify individual laser beams in the current scan as belonging to moving or static parts of the environment, finally we segment the current laser scan to extract objects from individual laser beams.

\section{A. Environment Mapping \& Localization}

We have used incremental mapping approach based on lidar scan matching algorithm to build a consistent local vehicle map. Based on occupancy grid representation, the environment is divided into a two dimensional lattice of rectangular cells and we keep track of probabilistic occupancy state for each cell of the grid. Environment mapping is essentially the estimate of posterior probability of occupancy for each grid cellgiven sensor observations at corresponding known poses. To know these pose values we need to solve the localization problem. A particle filter is used for this purpose. We predict different possible positions of the vehicle (one position of the vehicle corresponds to one particle) using the car-like motion model and compute the probability of each position (i.e, the probability of each particle) using the laser data and a sensor model.

\section{B. Moving \& Static Parts Distinction}

After a consistent local grid map has been constructed we classify the laser hit points in the current laser scan as dynamic or static by comparing them with the map constructed so far. The principal idea is based on the inconsistencies between observed free space and occupied space in the local map. Laser hits observed in free space are classified as dynamic whereas the ones observed in previously occupied place are static the rest are marked as unknown.

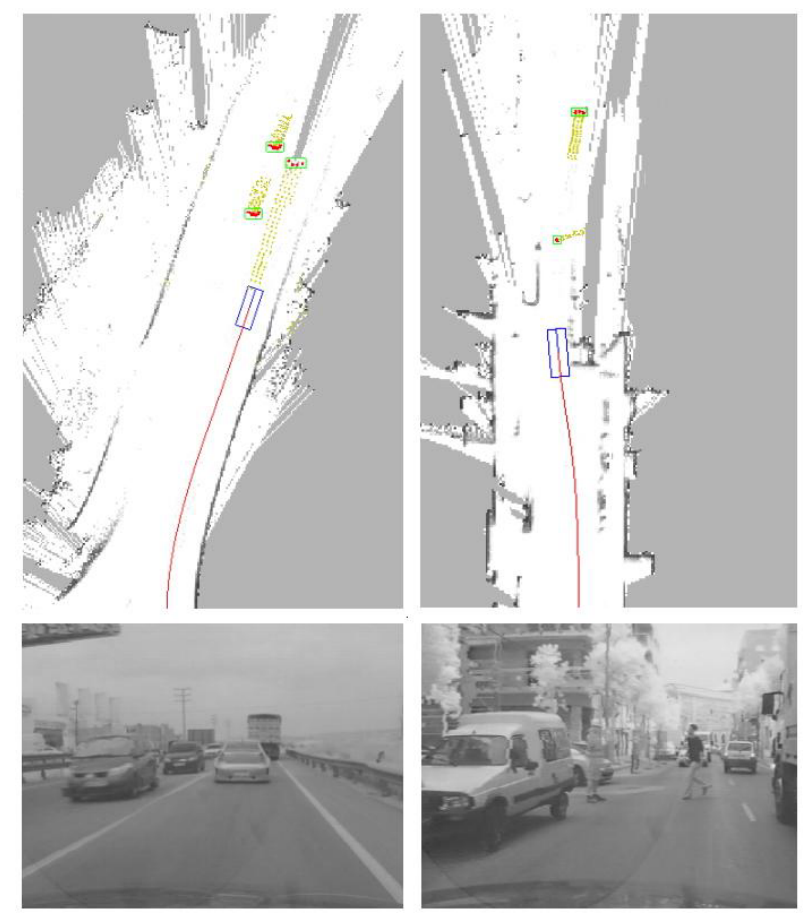

Fig. 3. Mapping and moving objects detection results. A bicycle and an oncoming moving car have been successfully detected.

\section{Laser Objects Extraction}

Objects are extracted from these laser hit points by a segmentation algorithm. Each segment found is considered as a separate object. An object that is marked as dynamic if at least one of its constituting laser point is classified as dynamic, otherwise it is considered as static. We also calculate the polar coordinates of center of gravity (centroid) of 
each segment using Cartesian coordinates of its constituting points. This information will be used to perform a polar fusion between lidar and stereo vision.

\section{Lidar Data Processing Output}

The output of lidar data processing consists of the local grid map and list of detected moving objects (we do not include the static objects in this list). Each object in this list is represented by its centroid and set of points corresponding to the laser hit points. Grid map is only used to display on the screen whereas list of dynamic objects is used further for fusion. An example of laser processing result is shown in the Figure 3.

\section{Stereo Vision Processing}

\section{A. Introduction}

The main roles of the stereo-vision sensor in an intersection driving assistance system are related to the sensing and perception in the front of the ego vehicle in a region up to $35 \mathrm{~m}$ in depth and a $70^{\circ}$ horizontal field of view. The current field of view was established as an optimum compromise between the maximum reliable depth range and the field of view. Static road and intersection environment perception functions are: Lane markings detection and 3D localization; Curb detection and 3D localization; Current and side lanes 3D model estimation based on lane delimiters (lane markings, curbs); Stop line, pedestrian and bicycle crossing detection and 3D localization; Painted signs (turn right, turn left, and go ahead) detection and 3D localization; Static obstacle detection, 3D localization and classification including parked vehicles, poles and trees. Dynamic road and intersection environment perception functions are: Preceding, oncoming and crossing vehicles detection, tracking and classification; Preceding, oncoming and crossing vulnerable road users detection, tracking and classification.

\section{B. Stereo sensor architecture for intersection assistance}

Based on the requirements analysis a two level architecture of a 6D stereo sensor was proposed [5] (Figure 4 and Figure ??). The low level architecture controls the image acquisition process and provides, after the sensor data processing, the primary information needed by the high level processing modules: $6 \mathrm{D}$ point information (3D position and 3D motion), ego motion estimation and intensity images at a rate of 20 frames per second. Using the rich output of the low-level architecture the two environment descriptions (structured and unstructured) are generated.

\section{Obstacle detection}

1) $3 D$ Points pre-processing: Using information from the digital elevation map the $3 \mathrm{D}$ points are classified according to their position with regard to the detected road/navigable area plane. Optical flow provides motion information for a subset of these points (corner points)

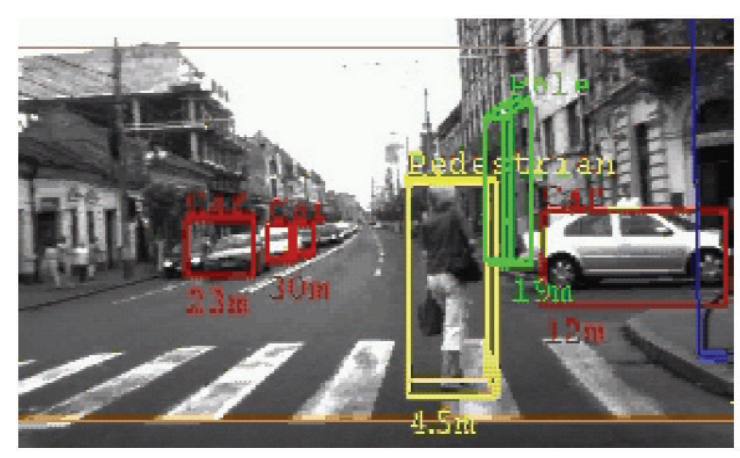

Fig. 5. Output of classification: the predicted class.

2) Obstacle detection: An improved obstacle detection technique was developed based on the fusion 3D position information with 3D motion information [7]. The obstacle detection algorithm extends the existing polar occupancy grid-based approach by augmenting it with motion information. The benefits gained from the integration of motion information are threefold. First, by use on at grid cell level, object boundaries are more accurately detected. Secondly, by exploiting motion at obstacle level, the obstacle's orientation is more accurately and naturally determined. And finally, each obstacle carries speed information, a valuable cue for tracking and classification. For non-stationary obstacles, motion can provide additional cues for orientation computation.The occupied areas are fragmented into obstacles with cuboidal shape without concavities and only with 90o convexities. There are 2 types of objects: 3D Non-Oriented and 3D Oriented. The Obstacles are represented as both oriented and non-oriented 3D boxes (cuboids) circumscribing the real obstacles in the scene. The Non-Oriented Obstacles are described by the minimum and maximum $\mathrm{X}, \mathrm{Y}$ and $\mathrm{Z}$ coordinates in the ego vehicle reference frame. The Oriented Obstacles are characterized by the $\mathrm{X}, \mathrm{Z}$ coordinates of the corners and the minimum and maximum $\mathrm{Y}$ coordinate.

3) Relevant obstacles classification: The goal of obstacle classification is to recognize the relevant objects in an intersection. We have identified three classes of relevant objects: Pedestrian, Pole, Car. A generic classification system able to recognize in real-time each of the three classes of objects has been developed [4] (Figure 5 ).

4) Obstacle representation: The obstacle are represented as cuboids carrying the following information:

- the cuboid's position, orientation and size,

- lateral and longitudinal speed,

- variance of the object center coordinates orientation and speed,

- tracking history (number of frames in which this object was previously seen),

- The detected obstacles are classified into: pedestrians, cars, poles and unknown.

To perform fusion between data from lidar and stereo vision objects, we project the objects detected by stereo vision processing onto the laser plane. 

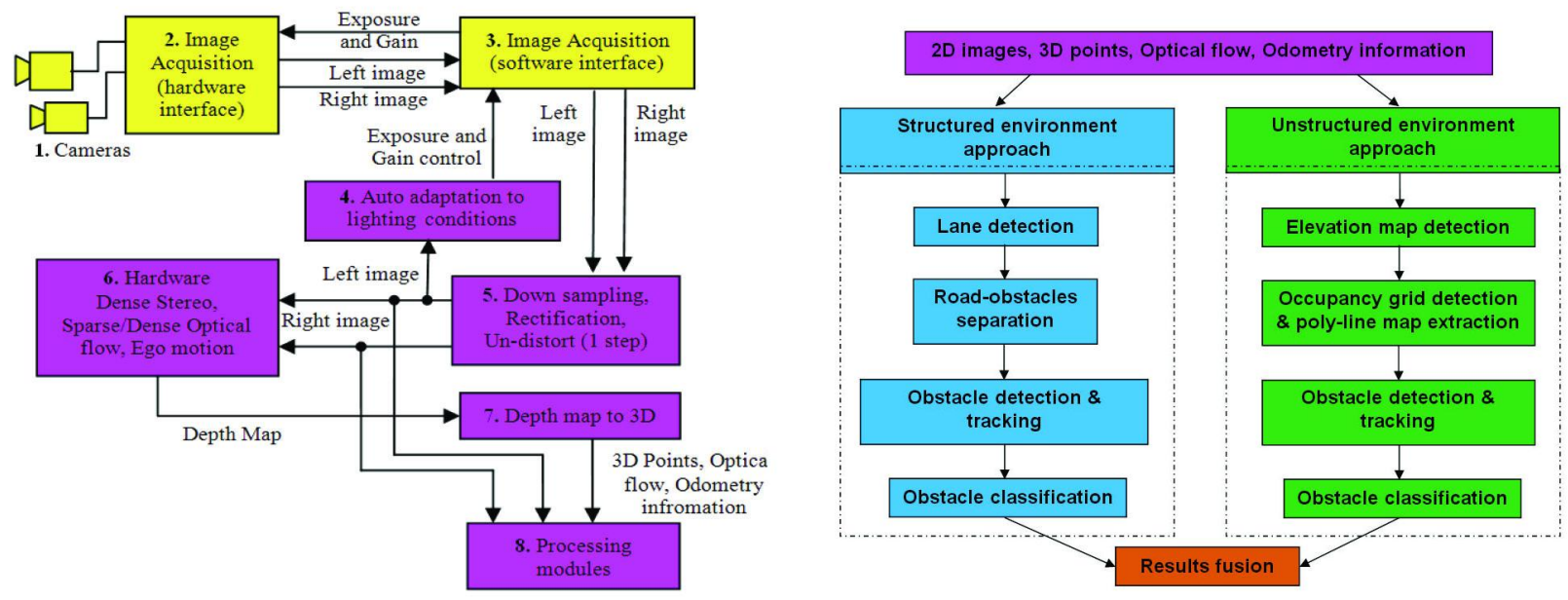

Fig. 4. Stereo-vision data processing: left) low-level architecture; right) high-level architecture

The output of stereo vision system consists of a list of objects with 2D position and classification information for each object. In the next step, we calculate the centroid of all the objects: it is the middle point of the front line segment of the object rectangle obtained after projection (this point gives better results than the center of gravity of this rectangle because laser readings also belong to the front end of the object). In the final step, we calculate the range and bearing of this centroid for each object from the origin of lidar frame of reference. So the object is represented as a point with range and bearing along with classification properties.

\section{LASER AND Stereo Vision Data Fusion}

Inputs to the fusion process are two lists of objects: list of dynamic objects detected by lidar processing and represented as centroid points, and list of dynamic objects detected by stereo vision processing represented as points along with classification information. We believe that an object detection level fusion between these two lists can complement each other thus giving more complete information about the states of objects in the environment. This fusion process consists of following two steps:

\section{A. Object association}

In this step we determine which stereo objects are to be associated to which lidar objects from the two object lists, using nearest neighbor technique. We have defined a distance threshold function based on the depth of the stereo object from the origin. Using this threshold value given by this function we associate the current stereo object to the nearest laser object lying within this threshold distance from the stereo object. We use this distance threshold function instead of a hardcoded value because depth uncertainty of stereo objects increases with the distance from the origin.

\section{B. Position information fusion}

This step works on the pair of objects associated with each other in the previous step and fuses their position (range and bearing) information. We model the position uncertainty using 2D guassian distribution for both objects. Suppose $P_{L}=\left[r_{L}, \theta_{L}\right]^{T}$ is the centroid position of laser object and $P_{V}=\left[r_{V}, \theta_{V}\right]^{T}$ is the centroid position of associated stereo vision object. If $X$ is the true position of the object then the probability that laser detects this object at point $P_{L}$ is given as:

$$
P\left(P_{L} \mid X\right)=\frac{e^{\frac{-\left(P_{L}-X\right)^{T} R_{L}^{-1}\left(P_{L}-X\right)}{2}}}{\sqrt{\left|R_{L}\right|^{8} 2 \pi}}
$$

and similar probability for stereo object is given as:

$$
P\left(P_{V} \mid X\right)=\frac{e^{\frac{-\left(P_{V}-X\right)^{T} R_{V}^{-1}\left(P_{V}-X\right)}{2}}}{\sqrt{\left|R_{V}\right|^{8} 2 \pi}}
$$

Here $R_{L}$ is the $2 X 2$ covariance matrix of range and bearing uncertainty calculated from the uncertainty values provided by the vendor. Whereas $R_{V}$ is the covariance matrix for stereo vision and depends on the depth of the object from origin In general range and bearing uncertainty for stereo objects is much higher than the corresponding objects detected by laser and increases with distance from the origin. Also, range uncertainty for stereo is greater than the bearing uncertainty in general. Using Bayesian fusion the probability of fused position $P$ is given as:

$$
P(P \mid X)=\frac{e^{\frac{-(P-X)^{T} R^{-1}(P-X)}{2}}}{\sqrt{|R|^{8} 2 \pi}}
$$

where $P$ and $R$ are given as:

$$
P=\frac{P_{L} / R_{L}+P_{V} / R_{V}}{1 / R_{L}+1 / R_{V}}
$$

and

$$
1 / R=1 / R_{L}+1 / R_{V}
$$

respectively.

The result of this fusion process is a new list of fused objects. This list also has all the laser objects which could not be associated with stereo objects and all the stereo 
objects which could not be associated with some laser objects. We keep unassociated stereo objects because they may correspond to dynamic objects which may not have been detected by laser either because they may are occluded or they may are transparent for laser.

\section{Fusion Output}

The output of fusion process consists of fused list of objects. For each object we have position (centroid) information, dynamic state information, classification information and a count for number of sensors detecting this object. For each fused object we also have a pointer to the original laser or stereo object to use segment or rectangle information while displaying the tracked object.

\section{TRACKING}

In general, the multi objects tracking problem is complex: it includes the definition of tracking methods, but also association methods and maintenance of the list of objects currently present in the environment. Bayesian filters are usually used to solve tracking problem. These filters require the definition of a specific motion model of tracked objects to predict their positions in the environment. Using the prediction and observation update combination, position estimation for each object is computed. In the following we explain the components of our tracking module.

\section{A. Data Association}

This step consists of assigning new objects of fused list to the existing tracks. Since in the current work we are more concerned with tracking multiple objects in an intersection like scenario so it is important to choose a more effective technique of data association. In an intersection scenario there may be many objects moving in different directions. They may be crossing or wating to cross in a direction perpendicular to the oncoming vehicles, for example a vehicle waiting to turn left etc. We have used MHT [8] approach to solve the data association problem. An important optimization that we have achieved here due to fusion process mentioned above is related to classification information provided by stereo vision. While generating hypotheses we ignore all those hypotheses which involve objects from different classes. For example a hypothesis trying to involve a pedestrain with a vehicle in a track will be ignored, this significantly reduces the number of hypotheses. To further control the growth of tracks trees we need to use some pruning technique. We have chosen the N-Scans pruning technique to keep the track trees to a limit of $\mathrm{N}$.

\section{B. Track Management}

In this step tracks are confirmed, deleted or created using the m-best hypotheses resulting from the data association step. New tracks are created if a new track creation hypothesis appears in the m-best hypothesis. A newly created track is confirmed if it is updated by objects detected in current frames after a variable number of algorithm steps (one step if the object was detected by both laser and stereo vision otherwise in three steps). This implies that the spurious measurements which can be detected as objects in the first step of our method are never confirmed. To deal with nondetection cases, if a non-detection hypothesis appear (which can appear for instance when an object is occluded by an other one) tracks having no new associated objects are updated according to their last associated objects and for them next filtering stage becomes a simple prediction. In this way a track is deleted if it is not updated by a detected object for a given number of steps.

\section{Filtering}

Since in an intersection like scenario there may be different types of objects (vehicles, motor bikes, pedestrains etc) moving in different directions using different motion modes, a single motion model based filtering technique is not sufficient. To address the tracking problem in this scenario we have used an on-line adapting version of Interacting Multiple Models (IMM) filtering technique. The details of this technique can be found in our other published work [9]. We have seen that four motion models (constant velocity, constant acceleration, left turn and right turn) are sufficient to successfully track objects on an intersection. We use four Kalman filters to handel these motion models. Finally the most probable trajectories are copmuted by taking the most probable branche and we select one unique hypothesis for one track tree.

\section{Tracking Output}

The output of tracking process consists of position and velocity information of ego vehicle alongwith a list of tracks. A track is a moving object with its position, orientation, velocity and classification information as well as a reference to its instance in the previous frame.

\section{RISK ASSESSMENT}

The risk assessment module provides an evaluation of the risk of a potential collision between the host vehicle and the objects that may be present in the driving environment. Our approach follows the work previously presented in [1]. This evaluation consists in the prediction of the environment for the future time instants and quantification of the risk associated to the detected collision situations: potential future collisions. It is considered that the driver has full control of the host vehicle and that the future driver behavior is unknown. The risk assessment is performed in the following sequential steps:

- scenario interpretation

- trajectory prediction

- collision detection

- risk quantification

- risk management

\section{A. Scenario interpretation}

The scenario interpretation consists of a representation of the current host vehicle state and local map of the surrounding environment composed of dynamic and static 
objects. This interpretation, that in most cases is incomplete and not very accurate, will influence the performance of the risk assessment. The host vehicle state provides information about the position, heading, steering angle, velocity, acceleration, yaw rate of the host vehicle. The dynamic objects can be of 2 types: vehicles and pedestrians. The information about the static and dynamic objects is limited and it leads to some assumptions that will influence the trajectory prediction process:

- The objects of type vehicle keep current speed and direction: no information about steering angle, acceleration, yaw rate or blinkers is provided by the high level fusion or communications.

- The host vehicle and other dynamic objects trajectories are not constraint to follow the road: there is no information about the static objects like the road geometry and lanes description.

\section{B. Trajectory prediction}

Given the current scenario interpretation the host vehicle state and dynamic objects are modeled and integrated in time to provide a projection of the future environment representation. This integration time consists in predicting the trajectories of the dynamic objects, including host vehicle, just by using the current scenario interpretation. The future driver behavior is unknown and will not be predicted although it may affect the future trajectories. A trajectory of a dynamic object is a temporal sequence of object states for the future time instants. For each object a trajectory predicted from the current time t0 until a given time horizon $\mathrm{t} 0+\mathrm{h}$ where $\mathrm{h}$ is the total prediction time. The modeling of the trajectories is done taking into account the object type, vehicle or pedestrian and associated information. The prediction of the vehicles trajectories, including the one of the host vehicle, is performed by using a bicycle dynamic model [3] integrated in time using the 4th order Runge-Kutta method that uses acceleration and steering rate commands as inputs. The initial vehicle state used to integrate the previous model is the one obtained at the time t0. Predict the movement of pedestrians is a substantially more difficult task [6] than vehicles. Since a pedestrian can easily change direction no assumptions are made regarding the direction of its movement. The pedestrian is then modelled as a circle with a predefined radius centred at the initially detected pedestrian position at time t0, that will increase its radius in time proportionally to its initially estimated speed.

\section{Collision detection}

The host vehicle and dynamic objects are represented as circles: the circle centre is given by the object position, and the circle radius is set according with the object type, at a given moment in time. The position uncertainty of the objects is represented by an increase of the circles radius in function of the estimated travelled distance by the object. A potential collision is detected when the host vehicle circle intersects at least one circle of the dynamic objects at the same moment in time. Figure 6 gives an illustration of this process.

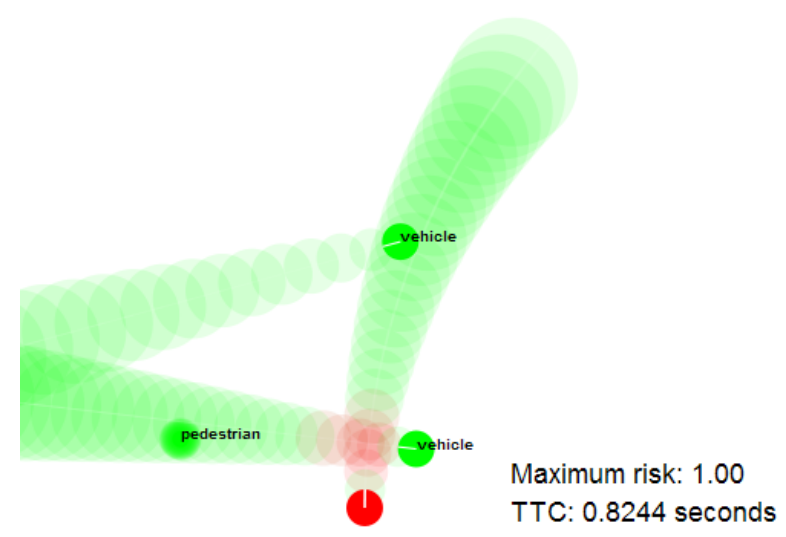

Fig. 6. Example of a potential collision between the host vehicle (red circle on the bottom) and another vehicle (green circle on the bottom right).

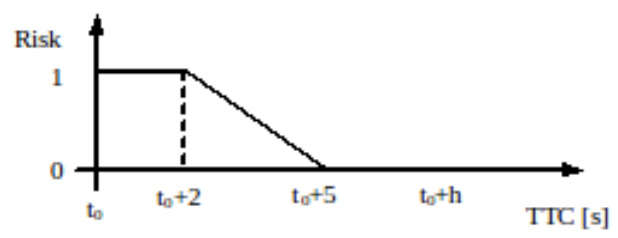

Fig. 7. Relation between TTC and risk indicator.

\section{Risk quantification}

The risk of collision is calculated for the nearest potential collision situation in time. For calculating this risk it is used the parameter time-to-collision (TTC) that corresponds to the duration between the current time t0 and the instant of when the first detected collision will occur. A consideration is taken that all objects keep their initial speeds until the moment of the collision. The TTC is an important parameter because it can be compared to the driver and vehicle reaction times and provide a collision risk indicator. In our implementation the obtained TTC is compared with the commonly used total reaction time of 2 seconds: driver $(1 \mathrm{sec})$ [10] and vehicle (1 $\mathrm{sec}$ ). The risk estimation performed until a predefined time horizon $\mathrm{t} 0+\mathrm{h}$ and the risk indicator is given by the relation shown in figure 7.

\section{E. Risk management}

Based on quantification of the collision risk two strategies can be adopted to avoid or minimize the potential accident: Information or warning: advices are provided to the driver through the appropriate HMI visual, audio, or haptic feedback to avoid or reduce the risk of accident. Intervention: the automation takes momentarily control of the vehicle to perform an obstacle avoidance or collision mitigation manoeuvre. In our implementation only visual information is provided to the driver with periodical estimation of the collision risk for the given scenario interpretation. 


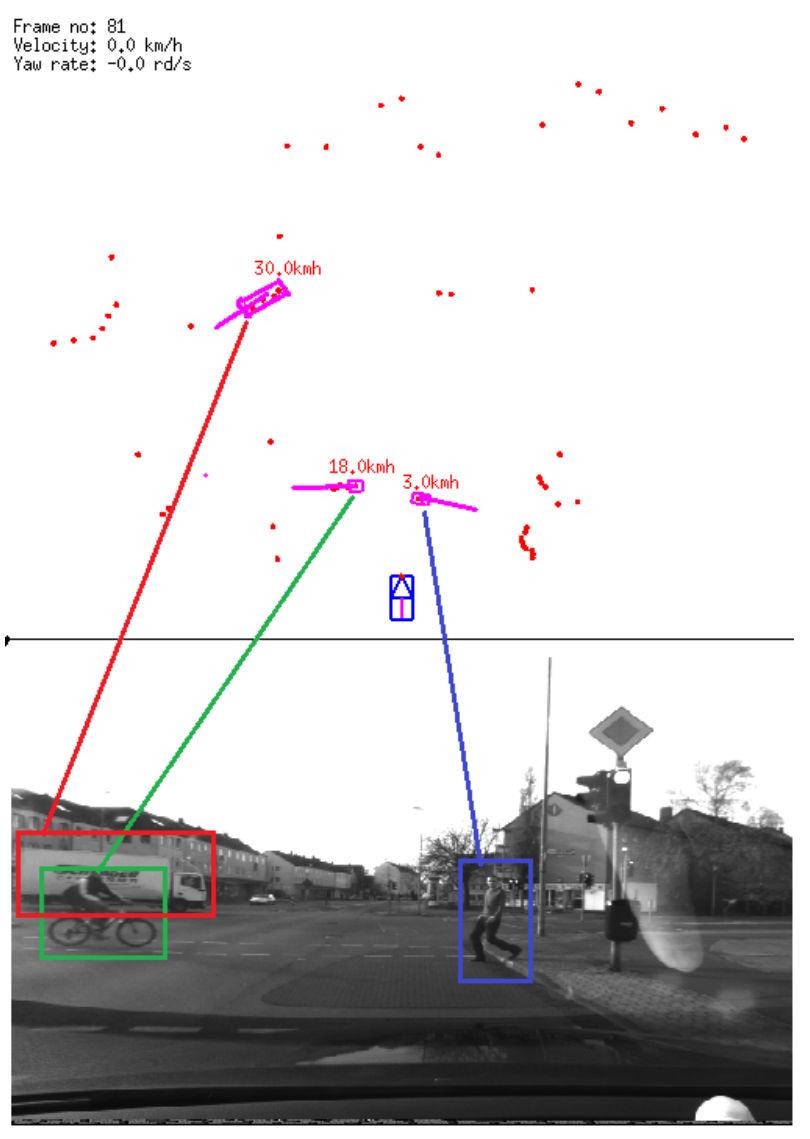

Fig. 8. Tracking results for a pedestrian and a cyclist.

\section{RESUlTS}

Examples of tracking results are shown in Figures 8 and 9 along with the images of corresponding scenarios. In Figure 8 is a situation at the intersection where the ego vehicle is waiting for the traffic signal, a cyclist and a pedestrian crossing the road in opposite directions are being tracked. In addition, a truck which is partially occluded by the cyclist is also well tracked. Figure 9 shows two cars crossing the intersection which are detected and tracked successfully.

\section{Conclusion}

In this paper, we describe our approach for the safety of vehicles at the intersection developed on the Volkswagen demonstrator. A complete solution to this safety problem including the tasks of environment perception and risk assessment are presented along with interesting results which could open potential applications for the automotive industry.

\section{ACKNOWLEDGEMENTS}

This work was conducted within the research project INTERSAFE-2 that is part of the 7th Framework Programme, funded by the European Commission. The partners of INTERSAFE-2 thank the European Commission for all the support.

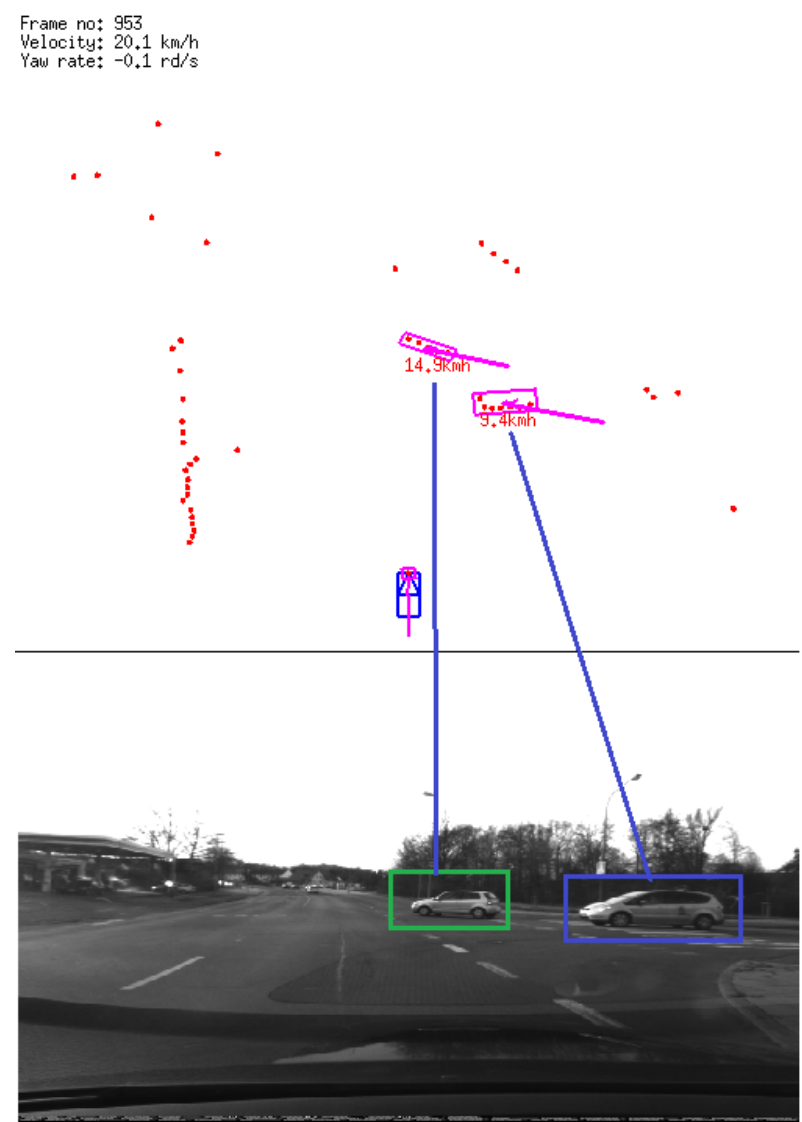

Fig. 9. Tracking results for two cars.

\section{REFERENCES}

[1] Samer Ammoun and Fawzi Nashashibi. Real time trajectory prediction for collsion risk estimation between vehicles. In IEEE International Conference on Intelligent Computer Communication and Processing, 2009.

[2] Q. Baig, TD. Vu, and O. Aycard. Online localization and mapping with moving objects detection in dynamic outdoor environments. In IEEE Intelligent Computer Communication and Processing (ICCP), Cluj-Napoca, Romania, August 2009.

[3] T.D. Gillespie. Fundamentals of Vehicle Dynamics, Society of Automotive Engineers. 1992.

[4] S. Nedevschi, S. Bota, and C. Tomiuc. Stereo-based pedestrian detection for collision-avoidance applications,. IEEE Transactions on Int, 10:380-391, 2009.

[5] S. Nedevschi, T. Marita, R. Danescu, F. Oniga, S. Bota, I. Haller, C. Pantilie, M. Drulea, and C. Golban. On Board $6 D$ Visual Sensors for Intersection Driving Assistance Systems. Advanced Microsystems for Automotive Applications, Springer, 2010.

[6] G. De Nicolao, A. Ferrara, and L. Giacomini. Onboard sensorbased collision risk assessment to improve pedestrians safety. IEEE Transactions on Vehicular Technology, 56(5):2405-2413, 2007.

[7] C. Pantilie and S. Nedevschi. Real-time obstacle detection in complex scenarios using dense stereo vision and optical flow. In IEEE Intelligent Transportation Systems, pages 439-444, Madeira, Portugal, September 2010.

[8] D. B. Reid. A multiple hypothesis filter for tracking multiple targets in a cluttered environment. Technical Report D-560254, Lockheed Missiles and Space Company Report, 1977.

[9] TD. Vu, J. Burlet, and O. Aycard. Grid-based localization and local mapping with moving objects detection and tracking. International Journal on Information Fusion, Elsevier, 2009. To appear.

[10] Y. Zhang, E. K. Antonsson, and K. Grote. A new threat assessment measure for collision avoidance systems. In IEEE International Intelligent Transportation Systems Conference, 2006. 